\title{
Prevalence of sarcopenia in multi ethnics adults and the association with cognitive impairment: findings from West-China health and aging trend study
}

Xiaolei Liu ${ }^{1,2}$, Lisha Hou ${ }^{1,2}$, Xin Xia ${ }^{1,2}$, Yang Liu ${ }^{1,2}$, Zhiliang Zuo ${ }^{1,2}$, Yan Zhang ${ }^{1,2}$, Wanyu Zhao ${ }^{1,2}$, Qiukui Hao ${ }^{1,2}$, Jirong Yue $e^{1,2}$ and Birong Dong ${ }^{1,2^{*}}$ (D)

\begin{abstract}
Background: Sarcopenia is a condition that is characterized by loss of muscle mass, muscle strength and muscle functional impairment with ageing. It is associated with poor health outcomes, premature death and a significant burden on the global health economy. The prevalence of sarcopenia in China is unknown since most of the studies are lack of uniform standard. The study was undertaken to study the prevalence of sarcopenia and the association with cognitive impairment among multi-ethnic adults aged 50 years old or older in western China.

Methods: We measured gait speed, handgrip strength and muscle mass by using bioelectrical impedance analysis (BIA) for all eligible participants and 4500 participants were eligible for the analysis. We defined sarcopenia using the diagnostic algorithm recommended by the Asian Working Group for Sarcopenia (AWGS). We assessed the participants' cognitive functions using the 10-item Short Portable Mental Status Questionnaire (SPMSQ). Relationships between sarcopenia and cognitive impairment were analyzed using univariate and multivariate analyses.
\end{abstract}

Results: Of 4500 participants (mean age $62.4 \pm 8.3$ years), 869 (19.31\%) adults were sarcopenia. 446 (9.9\%) participants were identified as having mild cognitive impairment, 144 (3.2\%) adults were identified as having moderate/severe cognitive impairment. After adjusting for age, gender, ethnics and other potential cofounders, cognitive impairment was found to be independently associated with sarcopenia with a dosage effect (mild cognitive impairment: odds ratio [OR]: 1.41, 95\% Cl 1.10-1.82; moderate/severe cognitive impairment: OR: 3.05, 95\% $\mathrm{Cl}$ 2.08-4.49). After gender stratification, the association between mild cognitive impairment with sarcopenia in male is not significant, while is still significant in female. While the association between moderate/severe cognitive impairment is independently associated with sarcopenia in both male and female.

Conclusions: The prevalence rates of sarcopenia, mild cognitive impairment, moderate/severe cognitive impairment among the communities aged 50 or older in western China were 19.31, 9.9 and 3.2\%, respectively. Cognitive impairment was significantly associated with sarcopenia with a dosage effect, especially in female.

Keywords: Sarcopenia, Aging, Western China, Multi-ethnic, Cognitive impairment

\footnotetext{
* Correspondence: birongdong123@outlook.com

${ }^{1}$ National Clinical Research Center for Geriatrics, West China Hospital, Sichuan University, No. 37, Guo Xue Xiang Renmin Nan Lu, Chengdu, Sichuan, China

${ }^{2}$ Geriatric Health Care and Medical Research Center, Sichuan University,

Chengdu, Sichuan Province, China
}

(c) The Author(s). 2020 Open Access This article is distributed under the terms of the Creative Commons Attribution 4.0 International License (http://creativecommons.org/licenses/by/4.0/), which permits unrestricted use, distribution, and reproduction in any medium, provided you give appropriate credit to the original author(s) and the source, provide a link to the Creative Commons license, and indicate if changes were made. The Creative Commons Public Domain Dedication waiver (http://creativecommons.org/publicdomain/zero/1.0/) applies to the data made available in this article, unless otherwise stated. 


\section{Background}

Sarcopenia was first named in 1989, an age-associated loss of skeletal muscle mass and function [1]. The 2010 European Working Group on Sarcopenia in Older People (EWGSOP) defines it as a type of progressive, extensive reduction in skeletal muscle mass, muscle strength and skeletal muscle dysfunction, leading to a decline in body function and quality of life, and even death [2]. It is generally characterized by decreased muscle mass and bone mass with aging, as well as skeletal muscle dysfunction, which in turn affects the normal physiological function and quality of life [3]. In 2016, sarcopenia was officially included in the ICD-10 disease code [2], marking the diagnosis of sarcopenia in the field of clinical medicine, which is considered as a disease with its unique characteristics. Besides, sarcopenia was considered as a mortality predictor in communitydwelling older adults, meaning that older adults with severe sarcopenia had an increased risk of death in the short term [4].

The incidence of sarcopenia reported in different countries varies greatly depending on the method of measurement, the population surveyed, and the diagnostic criteria. Prevalence rates using EWGSOP definition vary from 1 to $29 \%$ in elderly community-dwelling populations and from 14 to $33 \%$ in long-term care populations in western countries [5]. While the prevalence of sarcopenia in Asia is $2.5-45.7 \%$ using Asia Working Group for Sarcopenia (AWGS) definition [6]. However, many challenges remained to be solved in the future. Asia is made up of a great number of ethnicities. The majority of currently available studies have been published from eastern Asia. Therefore, more studies of sarcopenia in the south, southeastern, and western Asia should be promoted.

China is the most populous country in Asia with the world's largest aging population. By 2050, there will be 400 million Chinese citizens aged over 65 years old, and 150 million of whom will be over 80 years old [7]. Besides, China is a multi-ethnic country with 56 ethnicities. The prevalence of sarcopenia in multi-ethnic western China is unknown. Screening and assessment of sarcopenia may provide an opportunity for early detection, intervention, and monitoring of the most vulnerable Chinese elders to reduce morbidity, prevent disability, and enable more effective use of health care resources in minority areas. Besides, although the association between sarcopenia and adverse health outcomes has been described, the interrelationship between cognitive impairment and sarcopenia in older people remains a controversy.

In this study, we got the cross-sectional data from the West-China Health and Aging Trend (WCHAT), a longitudinal multi-center cohort research conducted in western China to assess the health and aging status in the multi-ethnic region. According to the diagnostic algorithm of AWGS, participants with low muscle mass as well as low muscle strength or physical performance were considered to have sarcopenia. We aimed to 1) evaluate the prevalence of sarcopenia according to the recommended criterion of the AWGS. 2) investigate the association between cognitive impairment and sarcopenia.

\section{Method \\ Participants}

The current research is a cross-sectional analysis obtaining baseline data of the WCHAT study collected from July to December in 2018. Data were collected from 4 provinces including Yunnan, Guizhou, Sichuan, and Xinjiang. All participants aged 50 years old or older were enrolled. Participants were recruited by convenience and asked verbally by the researchers about their willingness to take part in the study. Before investigation, informed consent was signed and obtained by each participant. Initially, we recruited 7536 community-dwelling multiethnic Chinese aged 50 and older in total. 4500 participants did the bioelectrical impedance analysis (BIA) which is available for the selection of sarcopenia (Fig. 1).

\section{Data collection}

All interviewers are medical students in Sichuan University and was trained for 2 days about how to collect questionnaire data through face to face, one-on-one personal interviews. Other anthropometric and bioimpedance measurements were collected by trained technicians.

\section{Sarcopenia screening}

Sarcopenia was measured by the recommended diagnostic algorithm of AWGS in which included three elements: muscle mass, muscle strength, and physical performance. Muscle mass was measured by the method of BIA and this has been verified reliability and validity in Chinese [8]. The BIA method adopted the INbody720 body composition instrument, which was widely used in the diagnosis of sarcopenia $[5,9,10]$. Previous research did not observe statistical difference between MRImeasured and BIA-derived SM [11]. And the cut-off value of appendicular skeletal muscle mass index (ASMI) was $7.0 \mathrm{~kg} / \mathrm{m}^{2}$ in men and, $5.7 \mathrm{~kg} / \mathrm{m}^{2}$ in women. While grip strength was used a dynamometer (EH101; Camry, Zhongshan, China) to test the muscle strength. During the test, subjects held the grip dynamometer with their dominant hand, stood upright, kept their feet naturally separated (shoulder-width apart) and their arms could naturally droop. At the beginning of the test, the subjects gripped the handle to their full capacity. Intermittent gripping, swinging of the arms, squats, or contact 


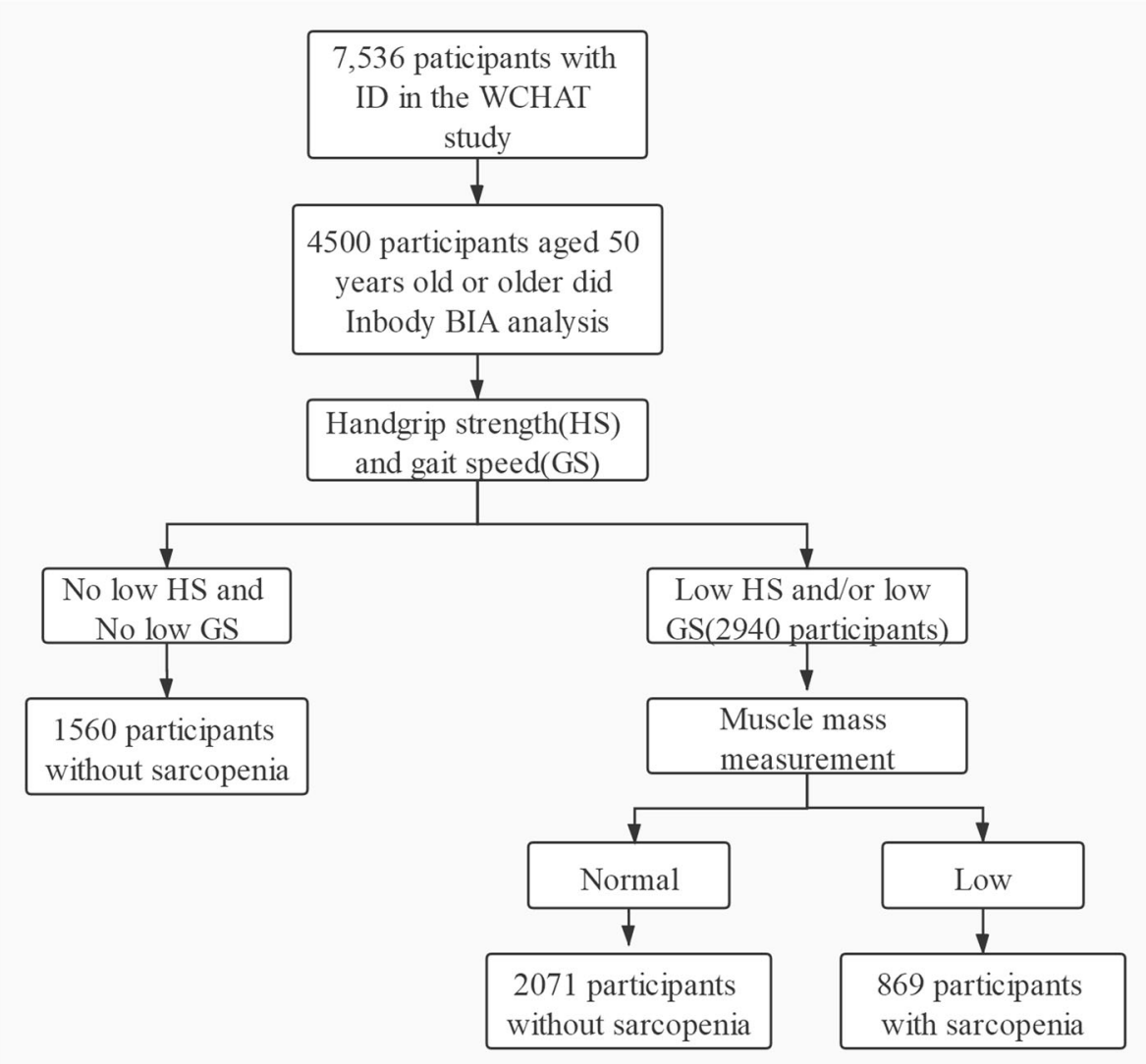

Fig. 1 Flow chart of study participants. Initially a total of 7536 community-dwelling multi-ethnics Chinese aged 50 or older were recruited, 4500 participants did bioelectrical impedance analysis (BIA) were analyzed in this study. Among them, 1560 participants without low gait speed and low grip strength, 2071 participants without low ASMI and 869 participants met the AWGS criteria of sarcopenia

with other body parts was prohibited. Test were performed on two independent occasions and the largest value was recorded. And the cut-off value was $26 \mathrm{~kg}$ in men and,18 kg in women [12]. Usual walking speed was measured. Subjects stood at the starting point and upon the starting command, walked forward at a normal pace to the 4-m line. The walking time was recorded using a kind of infrared sensor and the acceleration phase was strictly excluded. During the test, subjects wore common shoes, could use mobility aids, but could not be assisted. There were no time limits to the assessments and subjects could stop and rest if necessary. Sitting down was prohibited. And for sarcopenia definition, the cut-off value of gait speed was $0.8 \mathrm{~m} / \mathrm{s}$ [5].

\section{Demographic information}

Demographic information included age, gender, ethnics, marriage status, living status and education levels.

\section{Daily lifestyles and chronic diseases}

Smoking, alcohol drinking, and tea drinking habits were used to assess the daily lifestyles of the participants.
Smoking status was categorized as smoking and no smoking. Alcohol and tea drinking status was categorized as drinking and no drinking. Sleep quality was assessed using Pittsburgh sleep quality index (PSQI). Scores $>5$ are considered as poor self-reported sleep quality which was sensitive in distinguishing good vs. poor sleepers in Chinese [13]. Indoor housework and outdoor housework were also asked by the interviewers. A medical history of chronic disease was self-reported by the participants or their caregivers. These disease conditions included hypertension, coronary heart disease, chronic obstructive pulmonary disease, osteoarticular disease, tumor, stroke, diabetes mellitus, cataract, deaf and so on. Chronic diseases comorbidities were considered as having two or more kinds of chronic diseases.

\section{Cognitive function, depressive symptoms and anxiety status}

Cognitive status was measured using a 10-item Short Portable Mental Status Questionnaire (SPMSQ). For SPMSQ scoring, 0 2 indicated complete cognitive 
function, 3 4 indicated mild cognitive functional impairment, 5 7 indicated moderate cognitive function impairment, and 8 10 indicated severe cognitive function impairment. And this judgment was based on the educational level [14]. Depressive symptoms were assessed using the 15-item Geriatric Depression Scale (GDS-15). The scale, which contains 15 items that require only a yes/no answer, is the most widely used scale for the detection of depression. GDS-15 scores $\geq 5$ indicate depressive mood [15]. Anxiety status was assessed using Generalized Anxiety Disorder (GAD-7) questionnaire and the scores $\geq 5$ was considered as having anxiety status [16].

\section{Statistical analysis}

We test the normality of variables using $\mathrm{R}$ version 3.6.1. The measurement data is expressed by $\mathrm{X} \pm \mathrm{SD}$. For the normal distribution variables, the difference between the groups is compared by the independent sample $\mathrm{T}$-test and the count data is expressed in \%, using the $\chi^{2}$ test. We used $x 2$ test to determine whether the prevalence of cognitive impairment related factors was differed by sarcopenia status. Associations with a $p$-value of 0.1 or less in the univariate analysis were selected for the multiple regression analysis, which were used to estimate the odds ratio (OR) to identify associations between sarcopenia and cognition impairment after adjusting for potential confounders. A value of $\mathrm{P}<0.05$ (two-side) was considered to be statistically significant.

\section{Results}

Overall, we enrolled 4500 participants (1627 men and 2873 women) aged 50 years old or older in the study. The mean age of the group was 62.4 (SD:8.3) years. Most of the participants were female (63.8\%). 869 (19.31\%) adults were sarcopenia, 446 (9.9\%) participants were identified as having mild cognitive impairment, 144 (3.2\%) adults were identified as having moderate/severe cognitive impairment. The prevalence of sarcopenia in male was 22.1 and $17.8 \%$ in female. The prevalence of sarcopenia in the age group of $<65,65-74,75-84$ and $\geq$ 85 were $11.2,26.5,50.5$ and $65.2 \%$, respectively. The prevalence of sarcopenia in different ethnic groups of Han, Zang, Qiang and Yi were 22.3, 18.2, 11.8 and $34.7 \%$, respectively. An overview of clinical and sociodemographic data, anthropometric measures, cognition, depression, lifestyle factors, and chronic diseases are given in Table 1. Compared to the subjects without sarcopenia, those with sarcopenia were on average older, lower educational degree, living alone, bad marriage status (windowed or divorced), poor sleep quality, lower physical activities (indoor housework or outdoor housework), more smoking, less drinking tea, chronic diseases comorbidities burden, higher prevalence of depression and cognitive impairment (Table 1). And the prevalence of different ethnics varies significantly. While drinking alcohol and the prevalence of anxiety status is not significant between sarcopenia group and non-sarcopenia group (Table 1).

Table 2 shows the association between sarcopenia and cognitive impairment using multiple logistic regression in three models. In model 1 which only adjusted age, sex and ethnics, cognitive impairment (mild: OR 1.49, 95\% CI 1.16-1.92, $P=.002$; moderate/severe: OR 3.41, 95\% CI $2.34-4.97, P<.001)$ was significantly associated with sarcopenia (Table 2). After adding adjusting the marriage status, living alone, life-style factors, physical activities and sleep quality in model 2 , results showed that sarcopenia was still significantly associated with mild cognitive impairment (OR: 1.45, 95\% CI, 1.12-1.87, $P=.004)$ and moderate/severe cognitive impairment (OR: 3.27, 95\% CI, 2.23-4.79, $P<.001$ ) (Table 2). And this is consistent in the fully adjusted model 3 after adding chronic diseases comorbidities and depressive status which showed sarcopenia was significantly associated with mild cognitive impairment (OR: 1.41, 95\% CI, 1.10-1.82, $P=.009$ ) and moderate/severe cognitive impairment (OR: 3.05, 95\% CI, 2.08-4.49, $P<.001$ ). Besides, we analyzed the association between three elements of sarcopenia and cognition function separately. And it shows that cognitive impairment was significantly associated with grip strength, gait speed and ASMI separately with a dosage effect. After gender stratification in fully adjusted model, mild cognitive impairment is not significantly associated with sarcopenia in male, but is still significant in female. While moderate/severe cognitive impairment in male has a higher odds ratio than female (male: OR: 4.84, 95\% CI, 2.0111.62, $P<.001$; female: OR: 2.64, 95\% CI, 1.71-4.10, $P<.001$ ) (Table 3$)$. After age stratification in fully adjusted model, moderate-severe cognitive impairment was significantly associated with sarcopenia in participants less than 85 years old. Mild cognitive impairment was only associated with sarcopenia less than 65 years old (Table 3).

\section{Discussion}

According to reports from different countries in Asia, the prevalence of sarcopenia varies from $7.8-35.3 \%$ depending on different diagnostic instruments, different age and living conditions of the study participants in each study [17]. In our study, the mean age is 62.4 ( $\mathrm{SD} \pm$ 8.3 ) and the prevalence of sarcopenia is $19.31 \%$, which was similar to a cohort study in Taiwan using the AWGS diagnostic criteria that the prevalence of sarcopenia was $21 \%$ in participants aged 75 years and older [18]. Another cohort study in northern Taiwan included 302 individuals (157 men and 145 women) aged 65 years 
Table 1 Comparisons of demographic characteristics, anthropometric measures, life-styles, chronic diseases, depression, and cognitive impairment of the study participants with and without sarcopenia. $(N=4500)$

\begin{tabular}{|c|c|c|c|c|}
\hline Characteristics & No Sarcopenia $n=3631$ & Sarcopenia $n=869$ & $T / x^{2}$ & $p$ value \\
\hline Age,year,mean( $( \pm S D)$ & $61.0(7.5)$ & $67.9(8.8)$ & 21.39 & $<.001$ \\
\hline $50 \leq$ Age $<65, \mathrm{No}(\%)$ & $2475(88.8)$ & $313(11.2)$ & 433.88 & $<.001$ \\
\hline $65 \leq$ Age $\leq 74$, No (\%) & $956(73.5)$ & $345(26.5)$ & & \\
\hline $75 \leq$ Age $\leq 84$, No (\%) & $192(49.5)$ & $196(50.5)$ & & \\
\hline $85 \leq$ Age, No (\%) & $8(34.8)$ & $15(65.2)$ & & \\
\hline \multicolumn{5}{|l|}{ Gender No (\%) } \\
\hline Male & $1268(77.9)$ & $359(22.1)$ & 12.41 & $<.001$ \\
\hline Female & $2363(82.2)$ & $510(17.8)$ & & \\
\hline Ethnics & & & 83.15 & $<.001$ \\
\hline $\mathrm{Han}, \mathrm{No}(\%)$ & $1506(77.7)$ & $431(22.3)$ & & \\
\hline Zang,No(\%) & 1009 (81.8) & $224(18.2)$ & & \\
\hline Qiang,No(\%) & $926(88.2)$ & $124(11.8)$ & & \\
\hline Yi,No(\%) & $139(65.3)$ & $74(34.7)$ & & \\
\hline Others,No(\%) & $56(76.1)$ & $17(23.9)$ & & \\
\hline Education,No(\%) & & & 49.17 & $<.001$ \\
\hline No formal education & $972(26.8)$ & $322(37.1)$ & & \\
\hline Elementary school & $1161(32.0)$ & $287(33.0)$ & & \\
\hline Middle school & $787(21.7)$ & $134(15.4)$ & & \\
\hline High school and above & $711(19.6)$ & $126(14.5)$ & & \\
\hline Marital status, No (\%) & & & 56.84 & $<.001$ \\
\hline Single & $23(0.7)$ & $8(1.0)$ & & \\
\hline Married & $2965(86.0)$ & $628(76.0)$ & & \\
\hline Divorced & $52(1.5)$ & $15(1.8)$ & & \\
\hline Widowed & 409 (11.9) & $175(21.2)$ & & \\
\hline \multicolumn{5}{|l|}{ Anthropometric measures } \\
\hline Grip strength, mean $( \pm S D)$ & $23.0(8.7)$ & $17.6(6.8)$ & 19.94 & $<.001$ \\
\hline Gait speed, mean $( \pm S D)$ & $0.88(0.3)$ & $0.74(0.3)$ & 13.41 & $<.001$ \\
\hline ASMI, mean $( \pm S D)$ & $6.8(0.9)$ & $5.7(0.7)$ & 40.14 & $<.001$ \\
\hline \multicolumn{5}{|l|}{ Life-styles } \\
\hline \multicolumn{5}{|l|}{ Living alone No(\%) } \\
\hline Yes & $153(4.2)$ & $57(6.6)$ & 8.67 & .003 \\
\hline No & 3478 (95.8) & $812(93.4)$ & & \\
\hline \multicolumn{5}{|l|}{ sleep quality No(\%) } \\
\hline$P Q S \mid>5$ & $1595(46.3)$ & $420(51.0)$ & 5.90 & .015 \\
\hline$P Q S I \leq 5$ & $1848(53.7)$ & $403(49.0)$ & & \\
\hline \multicolumn{5}{|l|}{ Drinking Tea, No (\%) } \\
\hline Yes & $1672(48.7)$ & $373(45.5)$ & 2.72 & .099 \\
\hline No & $1762(51.3)$ & $447(54.5)$ & & \\
\hline \multicolumn{5}{|l|}{ Drinking alcohol, No (\%) } \\
\hline Yes & $876(25.5)$ & $195(23.8)$ & 1.08 & .299 \\
\hline No & $2559(74.5)$ & $626(76.2)$ & & \\
\hline \multicolumn{5}{|l|}{ Smoking history No(\%) } \\
\hline Yes & $551(16.0)$ & $187(22.8)$ & 21.16 & $<.001$ \\
\hline
\end{tabular}


Table 1 Comparisons of demographic characteristics, anthropometric measures, life-styles, chronic diseases, depression, and cognitive impairment of the study participants with and without sarcopenia. $(N=4500)$ (Continued)

\begin{tabular}{|c|c|c|c|c|}
\hline Characteristics & No Sarcopenia $n=3631$ & Sarcopenia $n=869$ & $T / x^{2}$ & $p$ value \\
\hline No & $2885(84.0)$ & $633(77.2)$ & & \\
\hline \multicolumn{5}{|l|}{ Indoor housework No(\%) } \\
\hline No & $952(27.8)$ & $335(41.2)$ & 55.80 & $<.001$ \\
\hline Yes & $2472(72.2)$ & $478(58.8)$ & & \\
\hline \multicolumn{5}{|l|}{ Outdoor housework No(\%) } \\
\hline No & $1974(57.8)$ & $511(63.1)$ & 7.54 & .006 \\
\hline Yes & $1441(42.2)$ & $299(36.9)$ & & \\
\hline Number of chronic diseases & & & 4.92 & .027 \\
\hline 0 or 1 , No (\%) & $934(25.7)$ & $192(22.1)$ & & \\
\hline$\geq 2, \mathrm{No}(\%)$ & $2697(74.3)$ & $677(77.9)$ & & \\
\hline \multicolumn{5}{|l|}{ Depressive status, No (\%) } \\
\hline GDS-15<5 & $2816(77.6)$ & $639(73.5)$ & 6.36 & .012 \\
\hline GDS-15 $\geq 5$ & $815(22.4)$ & $230(26.5)$ & & \\
\hline \multicolumn{5}{|l|}{ Anxiety status, No (\%) } \\
\hline GAD-7<5 & $2950(81.2)$ & $708(81.5)$ & 0.02 & .877 \\
\hline GAD-7 $\geq 5$ & $681(18.8)$ & $161(18.5)$ & & \\
\hline Cognitive function, No (\%) & & & 92.59 & $<.001$ \\
\hline Complete cognitive function & $3023(88.1)$ & $637(77.7)$ & & \\
\hline Mild impairment, No (\%) & $331(9.7)$ & $115(14.0)$ & & \\
\hline Moderate-severe impairment, No (\%) & $76(2.2)$ & $68(8.3)$ & & \\
\hline
\end{tabular}

Note. Means \pm standard deviation was shown. Others = other ethnics including Zhuang, Manchu, Hui, Mongolia, Tujia ethnics. Data are shown using \% or mean (standard deviation). $P$ values were calculated with chi-squared tests and Student's t tests for categorical and continuous variables, respectively

and older, and the prevalence of sarcopenia in that study was $18.6 \%$ in elderly women and $23.6 \%$ in elderly men using BIA analysis [11]. A previous study in Chengdu found approximately 10\% community-dwelling elderly have sarcopenia in a number of 947 residents aged $\geq 60$ years old which is also estimated by the BIA analysis [19]. And the prevalence of sarcopenia was $12.3 \%$ in Chinese men and $7.6 \%$ in Chinese women aged 70 years and older in Hongkong estimated by dual-energy X-ray absorptiometry [20]. This study found a higher prevalence than Chengdu and Hongkong. The reason might be that most participants were living in the village that were poorer and have lower education level even through the mean age of our study is lower than the previous studies. This shows sarcopenia are happening younger in rural areas. And the difference may also be derived from the geographical and diagnostic criteria for sarcopenia and the difference in cognitive function diagnostic criteria. Besides, our study also found that advanced age with a dose response effect associated with sarcopenia and this is compatible with many other studies [20-22]. Moreover, our study found that the prevalence of sarcopenia in male is higher than in the female. This is not consistent with other previous studies which showed Chinese women may be more vulnerable to severe sarcopenia in old age than men [23]. The reason might be that the mean age of male is older than the female in our study. Besides, our findings revealed the prevalence of sarcopenia in different ethnic groups are different. This might be related with different cultural life-styles and diet habit. Also related with different composition of age and gender. Further research of the ethnic groups and sarcopenia could be done in the future.

Our findings revealed that sarcopenia was significantly associated with cognition impairment in the multiethnic western Chinese population, independent of age, gender, ethnics, marriage status, lifestyle factors, indoor or outdoor housework, sleep quality, chronic diseases comorbidities and depression status. This is compatible with several other studies that shows a positive relationship between cognitive impairment and sarcopenia [2426]. While in the EPIDOS cohort study, sarcopenia was not shown to be associated with cognitive impairment in elderly women [27]. Several possible mechanisms in many studies could explain this association. Firstly, cognitive impairment leads to reduced physical activity and dietary intake, which could trigger excessive muscle loss in older adults, accelerating sarcopenia [28]. Secondly, low-grade inflammation is considered to be associated with both sarcopenia and cognitive impairment. 
Table 2 Multiple regression analysis of sarcopenia and the three factors of sarcopenia (grip strength, gait speed, ASMI) with cognitive impairment among multi-ethnics in the west China communities. $(N=4500)$

\begin{tabular}{|c|c|c|c|}
\hline Sarcopenia & Model 1, OR(95\%Cl), $p$ value & Model 2, OR(95\%Cl), $p$ value & Model 3, OR(95\%Cl), $p$ value \\
\hline \multicolumn{4}{|l|}{ Cognitive impairment } \\
\hline No & 1.0(Ref) & 1.0(Ref) & 1.0(Ref) \\
\hline Mild & $1.49(1.16-1.92), .002$ & $1.45(1.12-1.87), .004$ & $1.41(1.10-1.82), .009$ \\
\hline Moderate-severe & $3.41(2.34-4.97),<.001$ & $3.27(2.23-4.79),<.001$ & $3.05(2.08-4.49),<.001$ \\
\hline \multicolumn{4}{|l|}{ Grip strength } \\
\hline \multicolumn{4}{|l|}{ Cognitive impairment } \\
\hline No & 1.0(Ref) & 1.0(Ref) & 1.0(Ref) \\
\hline Mild & $1.47(1.19-1.82),<.001$ & $1.41(1.14-1.75), .002$ & $1.38(1.11-1.71), .004$ \\
\hline Moderate-severe & $2.24(1.51-3.32),<.001$ & $2.11(1.42-3.14),<.001$ & $2.00(1.34-2.97), .001$ \\
\hline \multicolumn{4}{|l|}{ Gait speed } \\
\hline \multicolumn{4}{|l|}{ Cognitive impairment } \\
\hline No & 1.0(Ref) & 1.0(Ref) & 1.0(Ref) \\
\hline Mild & $1.50(1.22-1.86),<.001$ & $1.44(1.16-1.78), .001$ & $1.42(1.14-1.76), .001$ \\
\hline Moderate-severe & $1.76(1.22-2.56), .003$ & $1.66(1.14-2.42), .008$ & $1.60(1.09-2.33), .016$ \\
\hline \multicolumn{4}{|l|}{ ASMI } \\
\hline \multicolumn{4}{|l|}{ Cognitive impairment } \\
\hline No & 1.0(Ref) & 1.0(Ref) & 1.0(Ref) \\
\hline Mild & $1.33(1.05-1.68), .017$ & $1.31(1.04-1.67), .025$ & $1.29(1.02-1.64), .038$ \\
\hline Moderat-severe & $2.83(1.96-4.07),<.001$ & $2.74(1.89-3.96),<.001$ & $2.60(1.79-3.77),<.001$ \\
\hline
\end{tabular}

Note. Abbreviations: OR odds ratio, $\mathrm{Cl}$ confidence interval

Model 1: adjusted for age, sex and ethnics

Model 2: adjusted for age, sex, ethnics, marriage status, living alone, life styles (smoking, drinking tea), sleep quality, indoor housework and outdoor housework

Model 3: adjusted for age, sex, ethnics, marriage status, living alone, life styles (smoking, drinking tea), sleep quality, indoor housework, outdoor housework,

chronic diseases comorbidities and depressive status

Interleukin-6 (IL-6) and tumor necrosis factor- $\alpha$ (TNF$\alpha)$ have also been reported as inflammatory parameters, which are the important factors to lead to sarcopenia [29] and the development of cognitive impairment [30]. Third, excessive oxidative stress related to chronic diseases also play important roles in age-related muscle atrophy, interfering with the balance between protein synthesis and breakdown, causing mitochondrial dysfunction, inducing apoptosis, and lead to sarcopenia [31]. While products of oxidative and nitrosative stress accumulate with aging, which is also the main risk factor for cognition impairment [32]. In the end, cognitive impairment reinforces and emphasizes the neuronal changes in the central nervous system leading to changes in the levels and activity of neurotransmitters, which together with the inadequate distribution of oxygen to the brain, lead to a reduction in motor units and in the ability to maintain muscle activation, which might be related to sarcopenia [33]. Thus, there exists a close relationship between sarcopenia and cognitive impairment which share some common mechanisms.

Besides, our study found that the association between sarcopenia with moderate/severe cognitive impairment in male has a higher odds ratio than female which might be related to hormone regulation. And moderate/severe cognitive impairment is positively related to sarcopenia in women and men, while the mild cognitive impairment seems not be related to sarcopenia in male. And a recent study indicated significant associations of sarcopenia and physical frailty with cognitive decline was could be more easier to be found in females [34]. One study explained this sex differences may be partly due to systemic inflammation [35]. A larger sample prospective cohort studies are required to illustrate gender differences. Besides, after age group stratification, it shows that moderate/severe cognitive impairment was significantly associated with sarcopenia nearly in every age group, proving a close relationship between sarcopenia and cognitive impairment. While in the age group over 85 years old, the relationship was not significant anymore might be the reason that there were only 23 participants in that age group which might exist some bias.

Moreover, our study analyzed the association between cognitive impairment and the three elements in the fully regression model and it shows significant association in grip strength, gait speed and ASMI with a dosage effect separately. And this is consistent with recent studies. A study had shown that handgrip strength could be a 
Table 3 Association of cognitive impairment and sarcopenia among multi ethnics in the west China communities after gender and age stratification $(N=4500)$

\begin{tabular}{|c|c|c|}
\hline Variables & Adjusted OR (95\% Cl) & $P$ value \\
\hline \multicolumn{3}{|l|}{ Male } \\
\hline \multicolumn{3}{|l|}{ Cognitive impairment } \\
\hline Mild & $1.46(0.89 ; 2.37)$ & .132 \\
\hline Moderate-severe & $4.84(2.01 ; 11.62)$ & $<.001$ \\
\hline \multicolumn{3}{|l|}{ Female } \\
\hline \multicolumn{3}{|l|}{ Cognitive impairment } \\
\hline Mild & $1.39(1.02 ; 1.89)$ & .035 \\
\hline Moderate-severe & $2.64(1.71 ; 4.10)$ & $<.001$ \\
\hline \multicolumn{3}{|l|}{$50 \leq$ Age $<65$} \\
\hline \multicolumn{3}{|l|}{ Cognitive impairment } \\
\hline Mild & $1.88(1.27-2.76)$ & .001 \\
\hline Moderate-severe & $3.66(2.06-6.53)$ & $<.001$ \\
\hline \multicolumn{3}{|l|}{$65 \leq$ Age $\leq 74$} \\
\hline \multicolumn{3}{|l|}{ Cognitive impairment } \\
\hline Mild & $1.11(0.73-1.69)$ & 0.630 \\
\hline Moderate-severe & $2.17(1.17-4.02)$ & 0.014 \\
\hline \multicolumn{3}{|l|}{$75 \leq$ Age $\leq 84$} \\
\hline \multicolumn{3}{|l|}{ Cognitive impairment } \\
\hline Mild & $1.47(0.80-2.71)$ & 0.210 \\
\hline Moderate-severe & $3.56(1.31-9.63)$ & 0.013 \\
\hline \multicolumn{3}{|l|}{$85 \leq$ Age } \\
\hline \multicolumn{3}{|l|}{ Cognitive impairment } \\
\hline Mild & - & NS \\
\hline Moderate-severe & - & NS \\
\hline
\end{tabular}

Note. Adjusted odds ratios were computed by adjusting for age, ethnics, marriage status, life-style factors (smoking, drinking tea), living alone, sleep quality, indoor housework, outdoor housework, chronic diseases comorbidities, depressive status; $\mathrm{Cl}$ confidence interval, $O R$ odds ratio, NS no significant

means of monitoring progression of cognitive decline [36]. And some longitudinal studies found that those with slower walking speeds and a greater decline in speed over time were at greater risk of developing dementia and could predict transitioning from mild to severe cognitive impairment [37, 38]. However, for the element of ASMI, recent study found that Lowerextremity functioning, rather than ASMI, is closely related to multiple cognitive domains [39]. While low muscle mass was found to be predictors of long-term mortality in nonagenarian and centenarian women [40]. Whether ASMI could be used as a predictor of cognitive impairment required more studies.

This is a large study to assess sarcopenia defined by the AWGS criteria and its association with cognitive impairment in multi-ethnic western China, adjusting the analysis for relevant confounders, such as sociodemographic characteristics, marriage status, lifestyle factors, and chronic diseases comorbidities. Nonetheless, this study presents some limitations. It is derived from a cross-sectional study, in such a way that it is not possible to conclude the existence of a causal association between sarcopenia in the participants and the associated factors mentioned here, which is feasible in longitudinal designs. Although regression models were adjusted for many variables, residual confounding is still possible. In addition, chronic disease information, cognition, and depression adjustment come from self-reported questionnaires, and in spite that all the data in the questionnaires have been previously validated, this information should be treated with caution. In the end, we conducted a centralized investigation, not a household survey in which most of the participants who can come by themselves are relatively healthy people. As a result of it, there exists offset in the result of prevalence. However, despite these limitations, this study has enough samples for adjustment of the regression models to major confusion factors of clinical interests. Moreover, it presented similar results to those found in studies with a more robust methodology.

\section{Conclusions}

This study demonstrated that the prevalence of sarcopenia was $19.31 \%$ among 4500 multi-ethnic participants aged 50 years old or older in western China and sarcopenia was significantly associated with cognitive impairment with a dosage effect, especially in female. This is a large cross-sectional study in multi-ethnic western China which is significant to further study of sarcopenia in China and filled up the prevalence of sarcopenia in the western Asia which is important in promoting Asia sarcopenia research as Asia is made up of a great number of ethnicities. Moreover, considering the health consequences of sarcopenia is increasingly being recognized, further longitudinal study is required to explore the causal relationship between sarcopenia and cognitive impairment.

\section{Abbreviations \\ ASMI: Skeletal muscle mass index; AWGS: Asian working group for Sarcopenia; BIA: Bioelectrical impedance analysis; EWGSOP: European working group on sarcopenia in older people; GAD-7: Generalized anxiety disorder; GDS-15: 15-item Geriatric depression scale; IL-6: Interleukin-6; OR: Odds ratio; SPMSQ: Short portable mental status questionnaire; TNF- a: Tumor necrosis factor-a; WCHAT: West-China health and aging trend}

\section{Acknowledgements}

We thank all the volunteers for the participation and personnel for their contribution in the WCHAT study.

\section{Consent to publish}

Not applicable.

\section{Authors' contributions}

$X L$ designed the study and wrote the paper. $L H, X X, Y L, Z Z, Y Z, W Z$ take part in the interpretation of data. $\mathrm{QH}, J Y, \mathrm{BD}$ reviewed and edited the manuscript. All authors have read and approved the manuscript. 


\section{Funding}

Supported by 1.3.5 project for disciplines of excellence, West China Hospital, Sichuan University; National Key R\&D Program of China (2017YFC0840100 and 2017YFC0840101); Sichuan Science and Technology Program (2018TJPT0015, 2018JY0094). The funding body had no role in the design of the study and collection, analysis, interpretation of data or in writing the manuscript.

\section{Availability of data and materials}

The datasets generated and analyzed during the current study are not publicly available due to this is a newly database which has a lot of important information and we are applying some important projects based on this. But this dataset will be available 2 years later and is also available now from the corresponding author on a reasonable request.

\section{Ethics approval and consent to participate}

The current research was approved by the Ethical Review Committee of West China Hospital of Sichuan University with the committee's reference number 2017(445) and the registration number is ChiCTR 1800018895. Participants were recruited by convenience and asked verbally by the researchers about their willingness to take part in the study. Before investigation, informed consent was signed and obtained by each participant.

\section{Competing interests}

The authors report no conflicts of interest in this work.

\section{Received: 7 December 2019 Accepted: 10 February 2020}

\section{Published online: 17 February 2020}

\section{References}

1. Rosenberg IH. Sarcopenia: origins and clinical relevance. J Nutr. 1997;127(5 Suppl):990s-1s.

2. Cruz-Jentoft AJ, Jean Pierre B, Bauer JM, et al. Sarcopenia: European consensus on definition and diagnosis: report of the European working group on sarcopenia in older people. Age \& Ageing. 2010;39(4):412-23.

3. Fielding RA, Vellas B, Evans WJ, et al. Sarcopenia: an undiagnosed condition in older adults. Current consensus definition: prevalence, etiology, and consequences. International working group on sarcopenia. J Am Med Dir Assoc. 2011;12(4):249-56.

4. Bachettini NP, Bielemann RM, Barbosa-Silva TG, AMB M, Tomasi E, Gonzalez MC. Sarcopenia as a mortality predictor in community-dwelling older adults: a comparison of the diagnostic criteria of the European Working Group on Sarcopenia in Older People; 2019.

5. Cruz-Jentoft AJ, Francesco L, Schneider SM, et al. Prevalence of and interventions for sarcopenia in ageing adults: a systematic review. Report of the international sarcopenia initiative (EWGSOP and IWGS). Age \& Ageing. 2014:43(6):748-59.

6. Chen LK, Liu LK, Woo J, et al. Sarcopenia in Asia: consensus report of the Asian working group for sarcopenia. J Am Med Dir Assoc. 2014;15(2):95101.

7. Fang EF, Scheibye-Knudsen M, Jahn HJ, et al. A research agenda for aging in China in the 21st century. Ageing Res Rev. 2015;24(Pt B):197-205.

8. Wang H, Hai S, Cao L, Zhou J, Liu P, Dong BR. Estimation of prevalence of sarcopenia by using a new bioelectrical impedance analysis in Chinese community-dwelling elderly people. BMC geriatrics. 2016;16(1):216.

9. Tosato M, Marzetti $\mathrm{E}$, Cesari $\mathrm{M}$, et al. Measurement of muscle mass in sarcopenia: from imaging to biochemical markers. Aging Clin Exp Res. Feb 2017;29(1):19-27.

10. Cruz-Jentoft AJ, Bahat G, Bauer J, et al. Sarcopenia: revised European consensus on definition and diagnosis. Age Ageing. 2019;48(4):601.

11. Chien MY, Huang TY, Wu YT. Prevalence of sarcopenia estimated using a bioelectrical impedance analysis prediction equation in communitydwelling elderly people in Taiwan. J Am Geriatr Soc. Sep 2008;56(9):1710-5.

12. Landi F, Calvani R, Tosato M, et al. Age-Related Variations of Muscle Mass, Strength, and Physical Performance in Community-Dwellers: Results From the Milan EXPO Survey. J Am Med Dir Assoc. 2017:18(1):88 e17-88.e24.

13. Tsai PS, Wang SY, Wang MY, et al. Psychometric evaluation of the Chinese version of the Pittsburgh sleep quality index (CPSQI) in primary insomnia and control subjects. Qual Life Res. 2005;14(8):1943-52.
14. Pfeiffer E. A short portable mental status questionnaire for the assessment of organic brain deficit in elderly patients. J Am Geriatr Soc. 1975;23(10): 433-41.

15. Lim PP, Ng LL, Chiam PC, Ong PS, Ngui FT, Sahadevan S. Validation and comparison of three brief depression scales in an elderly Chinese population. Int J Geriatric Psychiatry. 2000;15(9):824-30.

16. Swinson RP. The GAD-7 scale was accurate for diagnosing generalised anxiety disorder. Evidence-based medicine. 2006;11(6):184.

17. von Haehling S, Morley JE, Anker SD. An overview of sarcopenia: facts and numbers on prevalence and clinical impact. J Cachexia Sarcopenia Muscle. Dec 2010;1(2):129-33

18. Lin CC, Lin WY, Meng NH, et al. Sarcopenia prevalence and associated factors in an elderly Taiwanese metropolitan population. J Am Geriatr Soc. Mar 2013;61(3):459-62.

19. Wang H, Hai S, Liu Y, et al. Prevalence of sarcopenia and associated factors in community-dwelling elderly populations in Chengdu China. Sichuan da xue xue bao. Yi xue ban = Journal of Sichuan University. Medical science edition. 2019;50(2):224-8

20. Lau EM, Lynn HS, Woo JW, Kwok TC, Melton $\sqcup$ 3rd. Prevalence of and risk factors for sarcopenia in elderly Chinese men and women. J Gerontol A Biol Sci Med Sci. 2005;60(2):213-6.

21. Yu R, Wong M, Leung J, Lee J, Auyeung TW, Woo J. Incidence, reversibility, risk factors and the protective effect of high body mass index against sarcopenia in community-dwelling older Chinese adults. Geriatr Gerontol Int. 2014;14(Suppl 1):15-28.

22. Lee JS, Auyeung TW, Kwok T, Lau EM, Leung PC, Woo J. Associated factors and health impact of sarcopenia in older chinese men and women: a crosssectional study. Gerontology. 2007;53(6):404-10.

23. Lu X, Chu H, Wang L, et al. Age- and sex-related differences in muscle strength and physical performance in older Chinese. 2019.

24. Burns JM, Johnson DK, Watts A, Swerdlow RH, Brooks WM. Reduced lean mass in early Alzheimer disease and its association with brain atrophy. Arch Neurol. Apr 2010;67(4):428-33.

25. Hsu YH, Liang CK, Chou MY, et al. Association of cognitive impairment, depressive symptoms and sarcopenia among healthy older men in the veterans retirement community in southern Taiwan: a cross-sectional study. Geriatr Gerontol Int. Feb 2014;14(Suppl 1):102-8.

26. Nishiguchi S, Yamada M, Shirooka H, et al. Sarcopenia as a risk factor for cognitive deterioration in community-dwelling older adults: a 1-year prospective study. J Am Med Dir Assoc. 2016:17(4):372 e375-378.

27. Abellan van Kan G, Cesari M, Gillette-Guyonnet S, et al. Sarcopenia and cognitive impairment in elderly women: results from the EPIDOS cohort. Age Ageing. 2013;42(2):196-202.

28. Roubenoff R. Exercise, sarcopenia, cognition, and mood. Nestle Nutrition workshop series. Clinical \& performance programme. 2002;6:151-9 discussion 160-152.

29. Beyer I, Mets T, Bautmans I. Chronic low-grade inflammation and agerelated sarcopenia. Curr Opin Clin Nutr Metab Care. 2012;15(1):12-22.

30. Licastro F, Pedrini S, Caputo L, et al. Increased plasma levels of interleukin-1, interleukin-6 and alpha-1-antichymotrypsin in patients with Alzheimer's disease: peripheral inflammation or signals from the brain? J Neuroimmunol. 2000;103(1):97-102.

31. Meng SJ, Yu LJ. Oxidative stress, molecular inflammation and sarcopenia. Int J Mol Sci. 2010;11(4):1509-26.

32. Mangialasche F, Polidori MC, Monastero R, et al. Biomarkers of oxidative and nitrosative damage in Alzheimer's disease and mild cognitive impairment. Ageing Res Rev. 2009;8(4):285-305.

33. Walston J, Hadley EC, Ferrucci L, et al. Research agenda for frailty in older adults: toward a better understanding of physiology and etiology: summary from the American Geriatrics Society/National Institute on Aging research conference on frailty in older adults. J Am Geriatr Soc. 2006;54(6):991-1001.

34. Ohta Y, Nomura E, Hatanaka N, et al. Female dominant association of sarcopenia and physical frailty in mild cognitive impairment and Alzheimer's disease. J Clin Neurosci. 2019;70:96-101.

35. Canon ME, Crimmins EM. Sex differences in the association between muscle quality, inflammatory markers, and cognitive decline. J Nutr Health Aging. 2011:15(8):695-8

36. Fritz NE, McCarthy CJ, Adamo DE. Handgrip strength as a means of monitoring progression of cognitive decline - a scoping review. Ageing Res Rev. 2017;35:112-23. 
37. Hoogendijk EO, Rijnhart JJM, Skoog J, et al. Gait speed as predictor of transition into cognitive impairment: Findings from three longitudinal studies on aging. Exp Gerontol. 2019;129:110783.

38. Hackett RA, Davies-Kershaw H, Cadar D, Orrell M, Steptoe A. Walking speed, cognitive function, and dementia risk in the English longitudinal study of ageing. J Am Geriatr Soc. Sep 2018;66(9):1670-5.

39. Ishii H, Makizako H, Doi T, Tsutsumimoto K, Shimada H. Associations of skeletal muscle mass, lower-extremity functioning, and cognitive impairment in community-dwelling older people in Japan. J Nutr Health Aging. 2019;23(1):35-41.

40. Wang H, Hai S, Liu Y, Liu Y, Dong B. Skeletal muscle mass as a mortality predictor among nonagenarians and centenarians: a prospective cohort study. Scientific Reports. 2019;9(1):2420.

\section{Publisher's Note}

Springer Nature remains neutral with regard to jurisdictional claims in published maps and institutional affiliations.

Ready to submit your research? Choose BMC and benefit from:

- fast, convenient online submission

- thorough peer review by experienced researchers in your field

- rapid publication on acceptance

- support for research data, including large and complex data types

- gold Open Access which fosters wider collaboration and increased citations

- maximum visibility for your research: over $100 \mathrm{M}$ website views per year

At BMC, research is always in progress.

Learn more biomedcentral.com/submissions 